\title{
Peripheral Nerve Blockade in a Patient with Mastocytosis
}

\author{
Christopher H. Bailey ${ }^{1}$, Kent P. Weinmeister ${ }^{2}$ \\ ${ }^{1}$ Mayo Medical School, Mayo Clinic College of Medicine, Rochester, Minnesota, USA \\ ${ }^{2}$ Department of Anesthesiology, Mayo Clinic College of Medicine, Scottsdale, Arizona, USA \\ Email: bailey.christopher@mayo.edu
}

Received 15 September 2014; accepted 26 March 2015; published 27 March 2015

Copyright (C) 2015 by authors and Scientific Research Publishing Inc.

This work is licensed under the Creative Commons Attribution International License (CC BY). http://creativecommons.org/licenses/by/4.0/

\begin{abstract}
Mastocytosis is a rare group of disorders with chronic and episodic mast cell release of mediators which can have systemic and cutaneous manifestations. Triggers of anaphylaxis include commonly used medications for anesthesia, analgesia, and muscle relaxation. There is little in the literature regarding local anesthesia in emergent surgery for patients with mastocytosis. This case details the use of a peripheral nerve blockade for multiple surgeries and pain control in a patient with biopsy-proven mastocytosis.
\end{abstract}

\section{Keywords}

Mastocytosis, Urticaria Pigmentosa, Regional Anesthesia, Supraclavicular Blockade

\section{Introduction}

Mastocytosis is a rare group of disorders with chronic and episodic mast cell release of mediators including histamine, heparin and leukotrienes as well as others [1] [2]. While this release normally protects individuals from infection via an inflammatory response, patients with mastocytosis experience massive mediator release, with deleterious effects.

Mastocytosis is characterized by 1) skin findings, 2) symptoms arising from mediator release, and 3) organ infiltration (in systemic forms of the disease). Darier's sign occurs with urticaria and erythema within approximately 5 minutes of scratching, or rubbing of skin or skin lesions with a large quantity of mast cells present [3].

The most common complaint is pruritis, commonly due to exposure to heat, stress, certain drugs, or physical irritation [4]. The most common manifestation of mastocytosis is urticaria pigmentosa (UP), small yellow-tan or reddish-brown macules or papules [5] (Table 1). 
Table 1. Mediators in mastocytosis and their effects.

\begin{tabular}{ll}
\hline \multicolumn{1}{c}{ Mediator } & \multicolumn{1}{c}{ Effect } \\
Histamine & $\begin{array}{l}\text { Pruritis, urticaria, gastric hypersecretion, bronchoconstriction, increased } \\
\text { vasopermeability, systemic hypotension }\end{array}$ \\
Heparin & $\begin{array}{l}\text { Local anticoagulation, osteopenia, osteoporosis } \\
\text { Proteases }\end{array}$ \\
Fysteinyl leukotrienes (LTD4, LTC4, LTE4) & procollagenase and tissue remodeling (degrade fibronectin) \\
Prostaglandins (PGD2) & $\begin{array}{l}\text { Increased vasopermeability, vasodilation, bronchoconstriction } \\
\text { Platelet activating factor }\end{array}$ \\
Cytokines (TNF-alpha, TGF-beta, Nerve growth factor) & $\begin{array}{l}\text { Activation of vascular endothelial cells, cachexia, fibrosis } \\
\text { Increased vasopermeability, vasodilation }\end{array}$ \\
Growth factors (IL-3, IL-5, IL-6) & $\begin{array}{l}\text { Mast cell and eosinophil proliferation, B cell proliferation with polyclonal } \\
\text { increase in immunoglobulins and paraproteins }\end{array}$
\end{tabular}

Adapted from UpToDate [6].

\section{Case Report}

A 51 year-old male presents to his allergist 1 day after being bit by his dog, for a follow-up appointment regarding his diagnosis of mastocytosis 2 years prior. The patient's right wrist has punctures on the dorsal and volar sides, proximal to the wrist crease. He has significant erythema and swelling, with fluctuance on the volar wound without discharge. There is lymphangetic streaking through the antecubital fossa to the proximal arm without palpable lymphadenopathy. The patient reports numbness and tingling of the hand. Upon examination by the allergist, he is instructed to go to the Emergency. Orthopedic hand surgery is consulted for evaluation of cellulitis, pain, numbness and tingling. He is given tetanus prophylaxis and admitted to the Hand Surgery Service for emergent surgery.

The patient's allergist and anesthesiology are consulted to discuss the patient and determine the best method of anesthesia for the patient. The patient has biopsy-proven urticaria pigmentosis diagnosed by dermatopathology at our institution. Through a detailed history and physical at the time of diagnosis, the allergist has determined that the patient's triggers include heat (flushing, rash, tachycardia), and physical factors (erythema). His symptoms originated approximately 3 years prior and have progressed over this time period. The patient reports nausea and vomiting with meperidine and morphine, however no testing to confirm true allergy has been performed to date. He has symptoms consistent with orthostatic hypotension, but has not been evaluated by tilt-table nor for POTS. He has a history of GERD, no other history of gastrointestinal symptoms. He is a lifetime non-smoker, and has not been diagnosed with asthma, COPD, TB or coccidioidmycosis. He is negative for anaphylaxis with meperidine, dilaudid, morphine or alcohol. Negative for syncope, outside of an isolated event as a teen during which he describes a loss of consciousness and subsequent "seizure". He is negative for a history of anemia, thrombocytopenia, or leukopenia. His surgical history includes multiple, bilateral knee surgeries, tonsillectomy, a broken arm, a broken hand, and a colonoscopy 6 months prior without evidence of anaphylaxis or complication. There is no information detailing the anesthetic plan for his previous procedures. The patient does express concern during his evaluation about potential anaphylaxis during general anesthesia. The patient's tryptase level before surgery is $6.2 \mathrm{ng} / \mathrm{mL}$. His medications on admission include Baclofen $10 \mathrm{mg}$-prn, clonazepamprn, EpiPen-prn, ammonium lactate lotion-prn, fexofenadine $180 \mathrm{mg}$ daily, hydromorphone $8 \mathrm{mg}$ prn, ranitidine $150 \mathrm{mg}$ daily, zolpidem $12.5 \mathrm{mg}$ daily. The anesthesiologist performs an examination revealing a thyromental distance, and a mouth opening within normal limits. He has normal neck flexion and extension, and is given a Mallampati score of II. Heart and lung examination, and mental status are normal. ASA physical status classification is Class 2.

Prior to the procedure, a 16 gauge IV is placed in the right hand, in addition to a 20 gauge IV in the antecubital fossa placed in the ED. Lactated ringers solution and normal saline are administered independently via these lines. The patient is premedicated prior to surgery at 19:30 with $200 \mathrm{mg}$ hydrocortisone sodium succinate, $50 \mathrm{mg}$ 
diphenhydramine, and $150 \mathrm{mg}$ ranitidine. The anesthesia team performed a supraclavicular nerve block of the right upper extremity under ultrasound guidance. Local anesthesia is provided with $1 \%$ lidocaine, a catheter is placed, and $15 \mathrm{~mL} 0.75 \%$ bupivicaine administered at 20:12. The patient was given $1.5 \mathrm{~g}$ ampicillin-sulbactam at 20:15, $2 \mathrm{mg}$ midazolam in two $1 \mathrm{mg}$ doses at 20:30 and 20:33, respectively, and an additional $10 \mathrm{~mL} 0.75 \%$ bupivacaine at 20:37—the start of the surgical procedure. The patient is not covered during the procedure, nor warmed with a "Bair Hugger" device.

The surgical team places a well-padded extremity tourniquet, and the arm is exsanguinated by gravity with the extremity tourniquet inflated to $250 \mathrm{mmHg}$. During the procedure, a right open carpal tunnel release, right flexortenosynovectomy of all digital flexor tendons, with debridement of dog bite involving the right dorsal wrist with removal of nonviable skin and subcutaneous fat. A large suction drain is placed and secured using nylon sutures.

During the procedure, at 20:53, $20 \mathrm{mg}$ propofol is administered. An additional $20 \mathrm{mg}$ propofol is given at 21:15. Surgical stop time was 21:37, and the patient is transferred to the PACU at 21:46 awake and in stable condition. Two days later, further surgical debridement and irrigation is performed. Diphenhydramine, hydrocortisone, midazolam, lidocaine (1\%) and propofol are used by the anesthesia team without complication during this second procedure.

Pain control following each procedure is achieved using $0.2 \%$ ropivacaine via the supraclavicular catheter, fentanyl and hydromorphone.

\section{Discussion}

Specifically concerning for anesthesiologists caring for patients with mastocytosis is the potential of mast cell mediator release to trigger anaphylaxis in patients. The cumulative prevalence of which has been reported to be between 22 and 49\% in adults [7]. The stimuli for degranulation can be unique in each patient, and is best elucidated with a careful history from the patient [8]-[13]. Potentially, medications, surgical procedures, alcohol, infections, emotional stress, insect stings and other toxic exposures, and physical factors like extremes in temperature, sudden temperature changes and spicy foods may trigger mast cell release of mediators. Medications such as opioids, iodinated contrast, vancomycin (and other antibiotics), NSAIDs and muscle relaxants have been associated as triggers in $18 \%$ - $25 \%$ of cases [7] [14]. Of these, muscle relaxants have been frequently associated with anaphylaxis, among these rocuronium and succinylcholine have been associated with the highest number of allergic reactions [7] [15]. Mivacurium and atracurium, as well as other drugs such as morphine, codeine, and thiopental should be avoided if other agents may be used to equal efficacy [7] [16]. Lower risk options of opioids include fentanyl, sufentanil, remifentanil, and alfentanil [15]. Midazolam, propofol, etomidate and ketamine are hypnotic drugs associated with a lower risk of reaction [7]. Cis-atracurium, pancuronium, and vecuronium have a lower incidence of perioperative reactions [7]. Fluran inhaled anesthetics have not been reported to be associated with anaphylaxis [15]. Of note, there is limited evidence to link local anesthesia to an increased risk with mastocytosis patients [7]. Although less than $1 \%$ of all drug reactions to local anesthetics are true allergic reactions, it is recommended to avoid ester local anesthetics such as procaine, chloroprocaine, tetracaine, and benzocaine. Anaphylactic reactions to amide local anesthetics such as lidocaine, mepivicaine, prilocaine, bupivacaine, levobupivacaine and ropivacaine are exceedingly rare [17]. Preservative-free formulations of these medications are recommended.

Anaphylaxis has been seen in both cutaneous and systemic forms of the disease. Flushing, syncope and vascular collapse may occur, as can GI symptoms such as pain, diarrhea, nausea, vomiting, peptic ulcer disease and bleeding are seen [18]. Abrupt hypotension due to iodinated contrast in a patient with urticaria pigmentosa has also been described in the literature [19]. Excessive bleeding associated with mastocytosis may be due to heparin release by mast cells [20]-[23].

Medications which have been previously well-tolerated may be used, but for medications which tolerance is unknown, a graded provocation test should be done. Premedication with anti-allergic drugs has been suggested. However, the value of this is largely unproven, and severe reactions may still occur [7]. The mainstay medication for a systemic drug reaction in patients with mastocytosis is epinephrine, while multiple drugs may be used adjuvantly.

\section{Conclusions}

The literature regarding elective and emergent surgery in patients with known mastocytosis is sparce. Much of 
the existing literature recommends the use of lower-risk medications in the perioperative space. Inhaled fluran anesthetics are associated with an extremely low risk of reactions, but this case represents the successful use of amide local anesthetic via catheter for peripheral nerve blockade during multiple surgeries and for pain control between each surgery.

This case highlights the need for providers to consider anaphylactoid and other reactions in patients with urticarial pigmentosa or cutaneous mastocytosis when being evaluated for emergent surgery. Prevention is the best way to avoid systemic reactions in these patients. Using low-risk medications, especially when considering muscle relaxants, analgesics and local anesthetics may reduce complications in this uncommon patient population.

\section{References}

[1] Valent, P., Sperr, W.R., Schwartz, L.B. and Horny, H.P. (2004) Diagnosis and Classification of Mast Cell Proliferative Disorders: Delineation from Immunologic Diseases and Non-Mast Cell Hematopoietic Neoplasms. Journal of Allergy and Clinical Immunology, 114, 3-12. http://dx.doi.org/10.1016/j.jaci.2004.02.045

[2] Akin, C. and Metcalfe, D.D. (2004) Systemic Mastocytosis. Annual Review of Medicine, 55, 419-432. http://dx.doi.org/10.1146/annurev.med.55.091902.103822

[3] Soter, N.A. (2000) Mastocytosis and the Skin. Hematology/Oncology Clinics of North America, 14, 537-555. http://dx.doi.org/10.1016/S0889-8588(05)70295-1

[4] Kettelhut, B.V. and Metcalfe, D.D. (1994) Pediatric Mastocytosis. Annals of Allergy, 73, 197-202.

[5] Caplan, R.M. (1963) The Natural Course of Urticaria Pigmentosa. Analysis and Follow-Up of 112 Cases. Archives of Dermatology, 87, 146-157. http://dx.doi.org/10.1001/archderm.1963.01590140008002

[6] http://www.uptodate.com/contents/image?imageKey=RHEUM\%2F52832\&topicKey=ALLRG\%2F4786\&rank=1\%7E 72\&source=see_link\&search=mastocytosis\&utdPopup=true

[7] Brockow, K. and Bonadonna, P. (2012) Drug Allergy in Mast Cell Disease. Current Opinion in Allergy and Clinical Immunology, 12, 354-360. http://dx.doi.org/10.1097/ACI.0b013e328355b7cb

[8] Alvarez-Twose, I., Bonadonna, P., Matito, A., et al. (2013) Systemic Mastocytosis as a Risk Factor for Severe Hymenoptera Sting-Induced Anaphylaxis. Journal of Allergy and Clinical Immunology, 131, 614-615. http://dx.doi.org/10.1016/j.jaci.2012.10.052

[9] Bridgman, D.E., Clarke, R., Sadleir, P.H., Stedmon, J.J. and Platt, P. (2013) Systemic Mastocytosis Presenting as Intraoperative Anaphylaxis with Atypical Features: A Report of Two Cases. Anaesthesia Intensive Care, 41, 116-121.

[10] Kim, D.C. and Horan, R. (2003) Anaphylaxis to Insect Sting Associated with Urticaria Pigmentosa. Allergy and Asthma Proceedings, 24, 175-178.

[11] Koide, T., Nakajima, T., Makifuchi, T. and Fukuhara, N. (2002) Systemic Mastocytosis and Recurrent Anaphylactic Shock. The Lancet, 359, 2084. http://dx.doi.org/10.1016/S0140-6736(02)08908-0

[12] Ludolph-Hauser, D., Rueff, F., Fries, C., Schopf, P. and Przybilla, B. (2001) Constitutively Raised Serum Concentrations of Mast-Cell Tryptase and Severe Anaphylactic Reactions to Hymenoptera Stings. The Lancet, 357, 361-362. http://dx.doi.org/10.1016/S0140-6736(00)03647-3

[13] Metcalfe, D.D., Ed. (1999) The Mastocytosis Syndrome. 5th Edition, McGraw Hill, New York.

[14] Weingarten, T.N., Volcheck, G.W. and Sprung, J. (2009) Anaphylactoid Reaction to Intravenous Contrast in Patient with Systemic Mastocytosis. Anaesthesia and Intensive Care, 37, 646-649.

[15] Mertes, P.M., Malinovsky, J.M., Jouffroy, L., Aberer, W., Terreehorst, I., Brockow, K., et al. (2011) Reducing the Risk of Anaphylaxis during Anesthesia: 2011 Updated Guidelines for Clinical Practice. Journal of Investigational Allergology and Clinical Immunology, 21, 442-453.

[16] Dewachter, P., Mouton-Faivre, C., Cazalaa, J.B., Carli, P., Lortholary, O. and Hermine, O. (2009) Mastocytosis and Anaesthesia. Annales Françaises d'Anesthésie et de Réanimation, 28, 61-73. http://dx.doi.org/10.1016/j.annfar.2008.09.022

[17] Hepner, D.L. and Castells, M.C. (2003) Anaphylaxis during the Perioperative Period. Anesthesia Analgesia, 97, 13811395. http://dx.doi.org/10.1213/01.ANE.0000082993.84883.7D

[18] Potier, A., Lavigne, C., Chappard, D., Verret, J.L., Chevailler, A., Nicolie, B. and Drouet, M. (2009) Cutaneous Manifestations in Hymenoptera and Diptera Anaphylaxis: Relationship with Basal Serum Tryptase. Clinical Experimental Allergy, 39, 717-725. http://dx.doi.org/10.1111/j.1365-2222.2009.03210.x

[19] Hosking, M.P. and Warner, M.A. (1987) Sudden Intraoperative Hypotension in a Patient with Asymptomatic Urticaria Pigmentosa. Anesthesia Analgesia, 66, 344-346. http://dx.doi.org/10.1213/00000539-198704000-00011 
[20] Arias, M., Villalba, C., Requena, I., Vazquez-Veiga, H., Sesar, A. and Pereiro, I. (2004) Acute Spinal Epidural Hematoma and Systemic Mastocytosis. Spine, 29, E161-E163.

[21] Kauhanen, P., Kovanen, P.T., Reunala, T. and Lassila, R. (1998) Effects of Skin Mast Cells on Bleeding Time and Coagulation Activation at the Site of Platelet Plug Formation. Thrombosis and Haemostasis, 79, 843-847.

[22] Koenig, M., Morel, J., Reynaud, J., Varvat, C. and Cathebras, P. (2008) An Unusual Cause of Spontaneous Bleeding in the Intensive Care Unit-Mastocytosis: A Case Report. Cases Journal, 1, 100. http://dx.doi.org/10.1186/1757-1626-1-100

[23] Sucker, C., Mansmann, G., Steiner, S., Gattermann, N., Schmitt-Graeff, A., Loncar, R., et al. (2008) Fatal Bleeding Due to a Heparin-Like Anticoagulant in a 37-Year-Old Woman Suffering from Systemic Mastocytosis. Clinical and Applied Thrombosis/Hemostasis, 14, 360-364. http://dx.doi.org/10.1177/1076029607309173 